\title{
Executing a One Health approach during a zoonotic outbreak response
}

\author{
Peter Woodward ${ }^{\star 1}$, Melissa Kretschmer ${ }^{1}$, Hayley Yaglom², Craig Levy ${ }^{1}$, Peter Peter \\ Mundschenk ${ }^{3}$, Anne Justice-Allen ${ }^{4}$, Ronald Klein ${ }^{1}$, Tammy Sylvester ${ }^{1}$ and Jigna Narang ${ }^{1}$
}

${ }^{1}$ Epidemiology, Maricopa County Department of Public Health, Phoenix, AZ, USA; ${ }^{2}$ Arizona Department of Health Services, Phoenix, AZ, USA; ${ }^{3}$ Arizona Department of Agriculture, Phoenix, AZ, USA; ${ }^{4}$ Arizona Game and Fish Department, Phoenix, AZ, USA

\section{Objective}

Demonstrate the utility of a One Health collaboration during a leptospirosis outbreak to expand outreach in human, environmental and animal health arenas.

\section{Introduction}

The One Health paradigm emphasizes cooperation and interdisciplinary collaboration to promote health and well-being among people, animals and the environment. Though the concept of One Health has been around since the 1800's, the phrase "One Health" was more recently coined, and projects are being developed globally under its sponsorship. Maricopa County Department of Public Health (MCDPH) has been working at a local level to enhance its One Health surveillance efforts and partnerships. This One Health partnership, comprised of representatives from the Arizona Department of Agriculture (ADA), Arizona Department of Health Services (ADHS), Arizona Game and Fish Department (AzGFD), Arizona Veterinary Medicine Association (AzVMA), Centers for Disease Control \& Prevention (CDC), MCDPH, Midwestern University (MWU) Veterinary School, and local veterinarians, was employed during a response to the recent emergence of leptospirosis in Maricopa County, Arizona.

Leptospirosis is a zoonotic bacterial disease typically prevalent in tropical regions, especially island countries or low-lying areas that flood. In the United States, CDC reports 100-200 human cases annually. Within the last five years, there have been two confirmed travel-associated human cases reported in Maricopa County. However, no locally acquired human or canine leptospirosis cases were reported.

Two separate clusters of canine leptospirosis were reported in Maricopa County in 2016; the first was detected in February among canines within a household. To increase awareness in the veterinary community, the AzVMA published an article summarizing the cluster. This article might have aided in the identification of a second larger cluster in November that involved multiple veterinary and boarding facilities throughout Maricopa County. Following both clusters, capacity increased for canine and human surveillance, laboratory testing, and environmental remediation, and discussions were initiated regarding wildlife testing through the efforts of the One Health team.

\section{Methods}

A coordinated review of canine medical records verified suspicion of leptospirosis within the exposed canine population. A CDC questionnaire was modified by the One Health team and facilities were visited to identify possible sources of canine infection.

A Knowledge, Attitudes and Practice (KAP) survey was distributed through the AzVMA to guide veterinary education efforts. Lecture series, educational materials, and health alerts were created with input from One Health agencies for physicians, veterinarians, dog boarding facilities, and owners.

CDC subject matter experts assisted in the implementation of a serosurvey of both dog owners and veterinary staff to determine if zoonotic transmission had occurred at the home, veterinary clinic or boarding facility. CDC laboratory testing provided leptospirosis speciation of canine urine specimens.

\section{Results}

Medical records were abstracted for 79 suspect leptospirosis canine cases and 48 owners were interviewed to assess their risk and exposure factors for their dogs. Prior to the visit, some facilities had already implemented self-directed infection control activities. No procedural gaps were identified at the four canine boarding facilities and veterinary clinics visited.

The KAP survey was completed by 216 Arizona veterinarians and technicians. Educational outreach included three AzVMA newsletter articles distributed to approximately 1,100 registered veterinarians, one fact sheet regarding the leptospirosis vaccine, and three factsheets targeting prevention and infection control messages for boarding facilities, veterinary clinics and the home. A three-part lecture series presented jointly by ADHS, ADA, MCDPH, and MWU was attended by approximately 150 veterinarians. A health alert about the possibility of leptospirosis human cases was distributed by MCDPH to healthcare providers.

Eighty-five dogs with either compatible symptoms or exposure were tested through the CDC laboratory, $68(80 \%)$ were positive. Canine testing revealed different leptospirosis species between the two clusters, suggesting it was unlikely that they had a common source of exposure. No zoonotic transmission was identified among the 118 people tested in the serosurvey.

\section{Conclusions}

Pre-existing connections between public health and animal health partners helped facilitate and expand laboratory testing, diagnosis, reporting, outbreak tracking and prevention. The serosurvey provided a novel opportunity to identify cases amongst exposed people and provided insight into zoonotic transmission. Information gained from the KAP survey provided a gap analysis in veterinary services and guided education efforts. Since July 2017, no new canine cases have been reported to public health. However, further studies to identify sources of transmission in wildlife are being developed. The collaborative efforts of multiple agencies culminated in a robust outbreak response and the strengthened processes and relationships can be leveraged for future emerging diseases.

\section{Keywords}

One Health; Outbreak; Leptospirosis

\section{*Peter Woodward}

E-mail: peterwoodward@mail.maricopa.gov 